\section{New Method for Viewing Root Surfaces}

\section{GLORIA JAMES KERRY, B.S., D.D.S., M.S.*}

IN ANY DISCUSSION of root preparation in periodontal therapy invariably root instrumentation emerges as the prominent factor which determines success or failure. Paramount for success in the treatment of periodontal disease is the complete removal of all calculus and bacterial extensions into cementum.

The biological significance of a smooth cemental surface has not been precisely defined. Calculus and plaque can form on any root surface no matter how smooth it feels clinically. The relationship between root surface roughness and accumulation of bacterial plaque appears to be one of facilitation. Clinical assessment of root surfaces has always had to depend on tactile discrimination using a sharp explorer. Although this instrument is basic in our armentarium, it cannot record ultrastructural differences. The explorer merely gives a clinical impression. In the laboratory however, the scanning electron microscope is capable of conveying a three-dimensional quality or sense of reality to root surfaces. As it achieves great depth of field and a wide range of magnification, it is ideally utilized for visualizing root surface topography. However, it is impossible with a black and white micrograph (just as it is with a black and white photograph) to distinguish various shades or grading in the gray zones. Often the images lack sharpness and do not yield any idea as to depth of surface depressions or elevations.

Described in the Smithsonian magazine ${ }^{1}$ in August 1973 was a technique for vividly bringing out hidden details in black and white photographs called "density slicing and color coding." The technique involved placing a photograph under a television scanning camera; the scanner then examined each tiny portion of the picture and determined its photogenic density. Then the electrical signal from the scanner was fed through an analyzer that converted each given slice of photographic density to a color. This provided a picture in which contrasts stood out brilliantly, unlike the various shades in the gray scale of the original photograph. The application of density slicing and color coding to aid in visualizing root surfaces seemed plausible as the exact depth of calculus and plaque as well as the concentration and flow patterns could be determined.

\section{Materials and Methods}

A sampling of photographs and micrographs of tooth surfaces was selected for density slicing and color coding.

\footnotetext{
* Department of Periodontics, The University of Michigan School of Dentistry, Ann Arbor, Michigan 48104, and private practice, 1427 David Whitney Building, Detroit, Michigan 48226.
}

The resultant photographs were produced by directly photographing the image on the screen of a color television monitor. Color synthesis was accomplished by means of the CVI Model 606 Video Quantizer.* The CVI Model 606 is an instrument which may be used to achieve unusual variations in the amplitude characteristics of analog input signals. The unit is intended primarily for the processing of video signals but may also be used with other forms of data in the DC to 5 megahertz frequency range. The 606 may be used to select from 1 to 16 separate "slices" of data at preselected amplitudes. Slicing thresholds may be adjusted by means of front panel controls to produce linear or nonlinear patterns.

\section{RESULTS}

Figure 1 shows the cemental surface of a tooth without periodontal disease $(\times 1000){ }^{2}$ Numerous projections with small areas representative of cementocyte lacunae can be seen. These projections appear to be uniform and in a regular pattern. Figure 5, after density slicing and color coding, brings forth the hidden details of the black and white micrograph $(\times 1000)$. Both figures illustrate that root surfaces when viewed at the ultrastructural level are certainly not smooth. Figure 2 is a micrograph of a periodontally diseased root surface $(\times 1000) .{ }^{2}$ Many irregular foreign particles are seen on the surface. These nodules vary in size and regularity and appear clustered together. They are clusters of bacterial colonies. Other rather amorphous areas in this micrograph are suggestive of aggregates of plaque. Figure 6 vividly picks up the variations in the surface topography of this periodontally diseased root surface after density slicing and color coding $(\times 1000)$.

Various surface results have been attributed to the different methods of root instrumentation. Wilkinson and Maybury ${ }^{2}$ have described root surfaces following ultrasonic instrumentation as appearing chipped and fractured. Their findings suggested that the ultrasonic instrument produced surface depressions and ridges and they implied that the instrument tip was "impaled" in the root surface. ${ }^{2}$ Figure 3 is a root surface which has been instrumented with an ultrasonic instrument, the Cavitron PllL or P11R $(\times 1000)$. Figure 7 is the root surface treated with the ultrasonic instrument but following density slicing and color coding, it vividly shows the ridges and depressions produced by the ultrasonic instrument $(\times 1000)$. The chipped effect of root surface topography following ultrasonic instrumentation is contrasted colorfully.

Many investigators have described hand curettes as being capable of leaving the tooth surface smoother than any other type of instrument. Figure 4 is a root surface which has been instrumented with a hand curette $(\times 1000)$. It is evident that hand instrumentation and ultrasonic instrumentation produce dissimilar patterns

\footnotetext{
* Video Quantizer 606, manufactured by Colorado Video, Incorporated. P.O. Box 928, Boulder, Colorado 80302.
} 


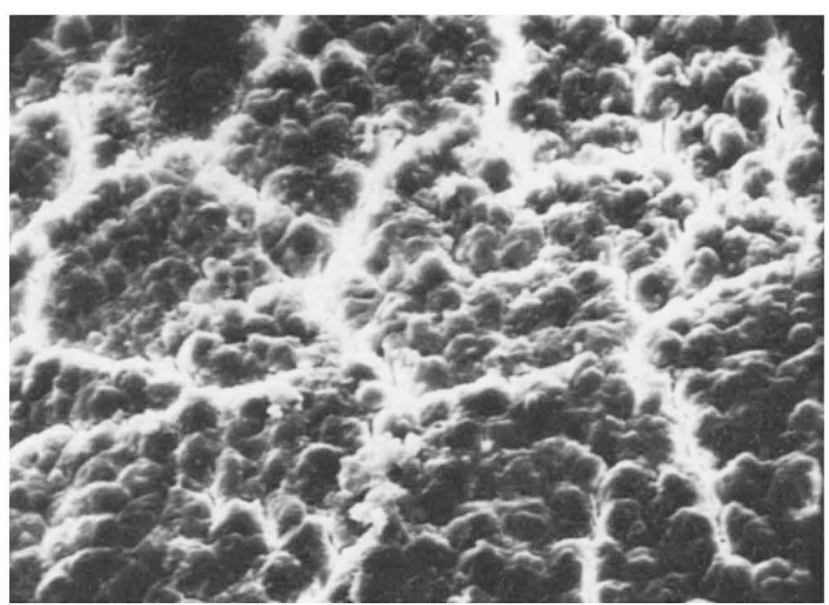

Figlre 1. Normal cemental surface-no periodontal disease. (Original magnification $\times 1000$.)

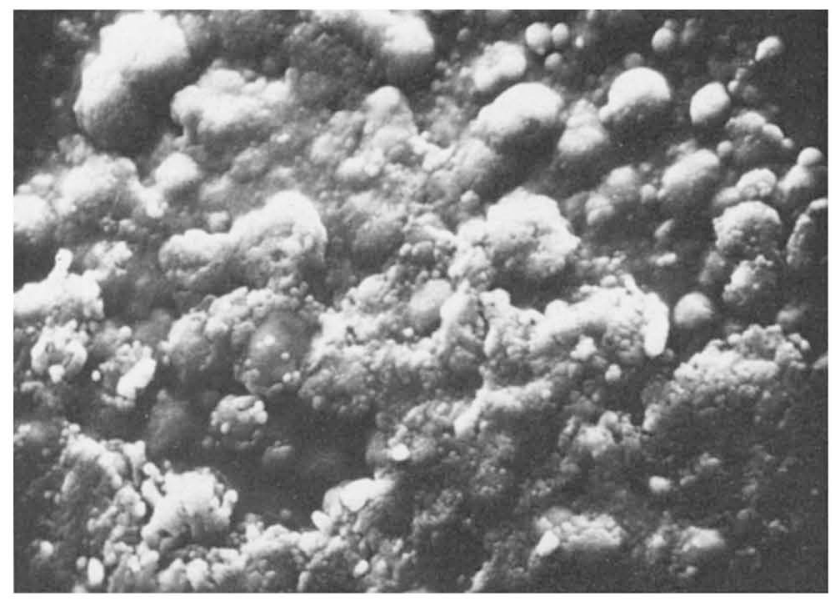

Figure 2. Periodontally diseased root surface. (Original magnification $\times 1000$.)

in the root surface. Figure 8 is the root surface instrumented with hand curettes following density slicing and color coding.

\section{Discussion}

Use of the technique density slicing and color coding affords an adjunctive method for viewing root surface topography. Utilizing all of the assets of the scanning electron microscope, this new technique affords a more definitive approach for the naked eye to distinguish the varying degrees of roughness or smoothness of root surfaces, the exact depth of calculus and the concentration and flow patterns of calculus and plaque. In root preparation, obtaining a hard and smooth surface is not as important as removing all calculus and bacterial extensions into root surface irregularities. Often in instrumentation, if one obtains a hard and smooth root surface, success is attributed to smoothness. However, it is probably the removal of calculus and bacterial extensions into the cementum that accounts for success. The benefits of complete removal of calculus, including decrease in inflammation, healing, and sometimes reattachment, are difficult to refute.
It is possible that any instrumentation of the root surface can result in scratching and grooving. Certainly all cutting instruments have the potential to damage tooth surface. Root surfaces are not as resistant to grooving and nicking as is enamel. Figure 9 illustrates the chatter marks and planing grooves which hand instruments can produce on the root surface $(\times 1000)$. This surface has been instrumented 15 strokes with a sharp curette. Hand instrumentation and ultrasonic instrumentation are both effective in removing foreign material from the root surfaces but viewing photographs at the ultrastructural level illustrates that any instrumentation alters the root surface. It appears that root planing with hand instruments and then burnishing with dull curettes produces the smoothest root surface. ${ }^{4,5,6}$ Figure 10 is a root surface which has been burnished with a dull curette $(\times 300)$. The instrument grooves have been planed smooth.



FIGURE 3. Root surface instrumented with Cavitron. (Original magnification $\times 1000$.)

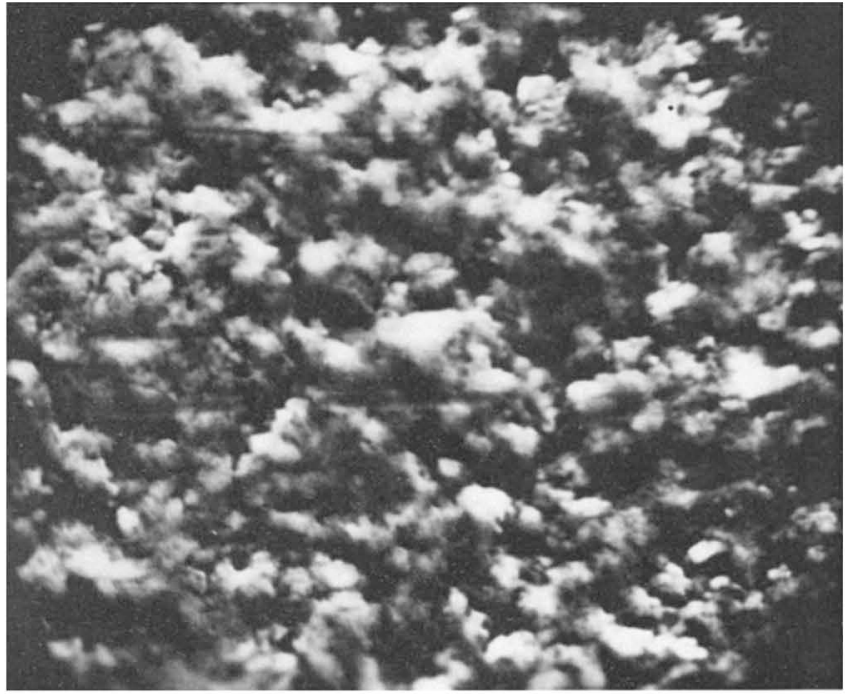

FIGURE 4. Root surface instrumented with hand curettes. (Original magnification $\times 1000$.) 



Figure 5. Normal cemental surface after density slicing and color coding. (Original magnification $\times 1000$.)

Figlre 6. Periodontally diseased root surface following density slicing and color coding. (Original magnification $\times 1000$.)

FIGURE 7. Root surface instrumented with ultrasonic instrument following density slicing and color coding. (Original magnification $\times 1000$.)

FIGURE 8. Root surface instrumented with hand curettes following density slicing and color coding. (Original magnification $\times 1000$.) FIGURE 9. Chatter marks and planing grooves produced by sharp curette. (Magnification $\times 1000$.)

FIGURE 10. Root surface burnished with dull curette. (Original magnification $\times 3000$.) 
Recently a new prophylaxis unit, the Orbison, has been introduced. This utilizes an air-driven turbine which produces an orbital motion at the working tip. In the hands of this investigator the Orbison is capable of removing supragingival calculus and plaque, but until there are improvements in the design, number and variation of the instrument tips, the Orbison is not clinically comparable to hand curettes or ultrasonic instruments in removing subgingival calculus.

It is evident that different methods of instrumentation produce dissimilar root surface topography. Whether or not there is a biological significance corresponding to the differences in root surface topography following the use of various methods of root surface instrumentation has not been definitely established.

\section{Summary and CONClusions}

A new technique, density slicing and color coding, was utilized in examining root surfaces. This technique proved to be an adjunct in evaluating tooth surfaces and appeared to have future dental application. Density slicing and color coding vividly portrays the root surface, the depth of depressions and the concentration and flow pattern of calculus, enhancing the ability of the naked eye to visualize root surface topography.

\section{ACKNOWLEDGMENTS}

Appreciation is expressed to Mr. Glen Southworth of Colorado Video, Incorporated, for his assistance on photography.

Appreciation is also expressed to Sandra L. Walker, R.D.H., B.S., Raymond F. Wilkinson, D.D.S., and Joseph F. Maybury, D.D.S., for their cooperation in furnishing black and white photographs.

\section{REFERENCES}

1. Edelson, Edward: Gaudy new way to see things you cannot see. Smithsonian (magazine) 4: 22, August 1973.

2. Wilkinson, Raymond F., and Maybury, Joseph E.: Scanning electron microscopy of the root surface following instrumentation. J Periodontol 44: 559, 1973.

3. Walker, Sandra L.: Evaluation of root planing by scanning electron microscopy. Thesis, University of Michigan, 1972.

4. Kerry, Gloria J.: Roughness of root surfaces after use of ultrasonic instruments and hand curettes. $J$ Periodontol 38: $340,1967$.

5. Green, E., and Ramfjord, S. P.: Tooth roughness after subgingival root planing. J Periodontol 37: 396, 1966.

6. Green, Edward: Root planing with dull and sharp curettes. J Periodontol 39: 3118, 1968.

\section{Abstracts}

The Structure of Subgingival Plaque in a Beagle Dog

Soames, J. V., and Davies, R. M.

J Periodont Res 9: 333, No. 6, 1974.

To determine the ultrastructure of bacterial deposits within the gingival crevice of the beagle dog, the maxillary canines and the second and third premolar teeth with their supporting bone and gingival tissues which showed clinical signs of gingivitis, were removed from a nine-month-old Beagle dog. Findings observed near the orifice of the crevice were gram-negative and gram-positive organisms, which in many areas appeared as microcolonies; deeper in the crevice the gram-negative filamentous predominated, more apically; gram-negative filamentous spirochaetes were observed, and beyond this zone, only spirochaetes which decreased at the most apical extents of the plaque. Coronally the spirochaetes were aligned parallel with their long axis vertical to the tooth surface. Since beyond the spirochaete zone the attachment of the epithelial junction was destroyed and since spirochaetes were the most apically located organisms found, it was suggested that they could be involved in the destruction of the epithelial attachment. Department of Oral Medicine, University of Manchester Dental Hospital, Bridgeford Street, Manchester, M15 5FH, England

SOME Aspects of Oral Immunology

Ferguson, J. W.

NZ Dent J 71: 16, January, 1975.

An outline of basic modern immunological concepts was given and the role of the immune response in certain oral diseases such as dental caries, oral cancer, recurrent oral ulceration, hypersensitivity, Sjögren's syndrome, chronic candidiasis and periodontal disease were discussed with reference to recent research. The immune mechanisms in periodontal disease were significantly involved in the initiation of gingival inflammation and in the subsequent breakdown of connective tissue and resorption of bone. 93 Stanley Point Road, Devonport, Auckland 9, New Zealand

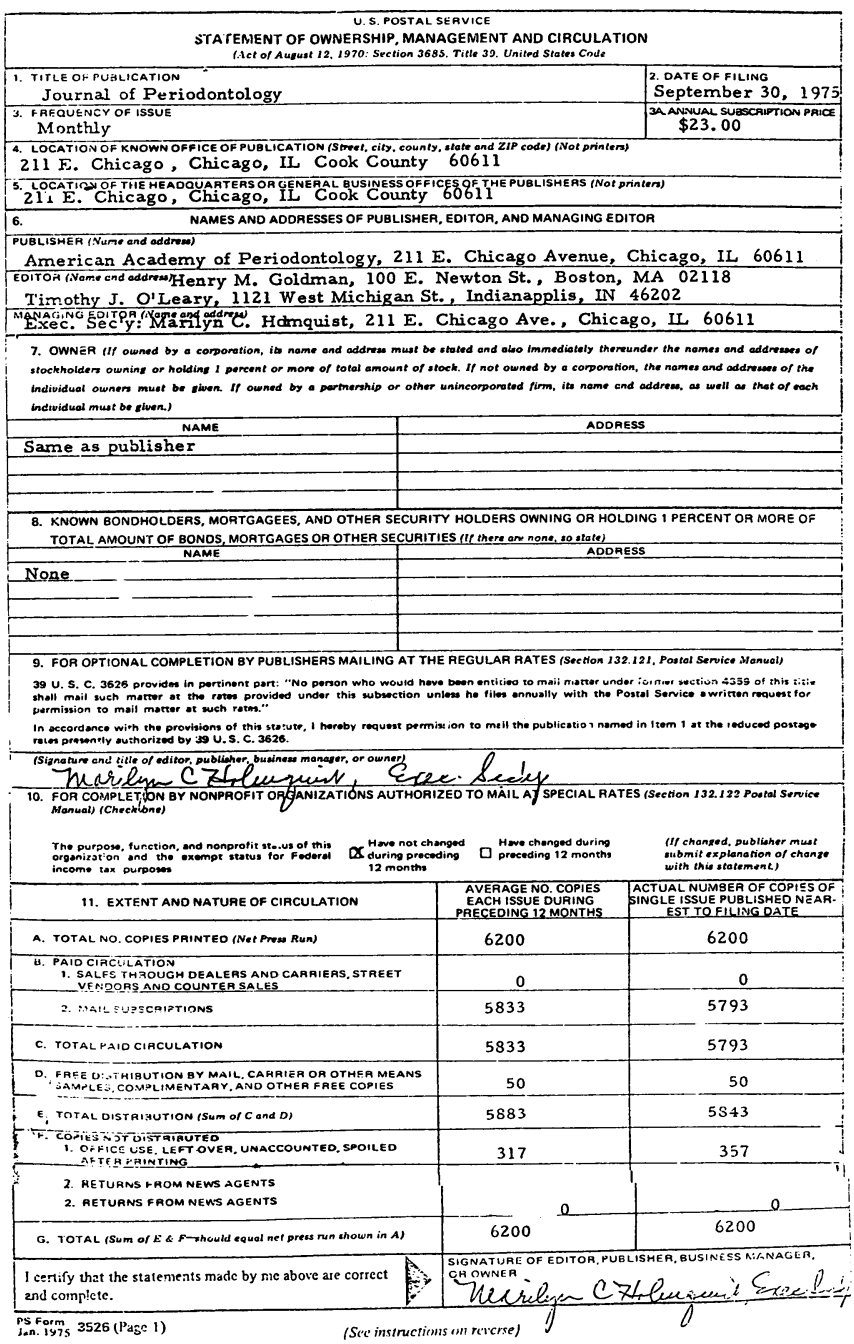

